\title{
PET/MRI is useful for early detection of pelvic insufficiency fractures after radiotherapy for cervical cancer
}

\author{
MAHO AZUMI ${ }^{1}$, MASUYO MATSUMOTO ${ }^{1}$, KAHO SUZUKI $^{1}$, RYOHEI SASAKI $^{2}$, \\ YOSHIKO UENO ${ }^{3}$, MUNENOBU NOGAMI ${ }^{3}$ and YOSHITO TERAI ${ }^{1}$ \\ Departments of ${ }^{1}$ Obstetrics and Gynecology, ${ }^{2}$ Radiation Oncology and ${ }^{3}$ Radiology, \\ Kobe University Graduate School of Medicine, Kobe, Hyogo 650-0017, Japan
}

Received June 12, 2021; Accepted August 25, 2021

DOI: $10.3892 / 01.2021 .13037$

\begin{abstract}
Radiotherapy (RT) is used to manage cervical cancer, and pelvic insufficiency fracture (PIF) is known as a late complication of RT. The present study identified risk factors for PIF after radiotherapy for cervical cancer, and investigated its incidence rate. It also considered the usefulness of positron emission tomography/magnetic resonance imaging (PET/MRI) in PIF diagnosis. A total of 149 patients with cervical cancer who received definitive or adjuvant RT with/without concurrent chemotherapy between January 2013 and December 2018 were investigated in the present study and followed up for more than one month after RT at Kobe University Hospital. The median follow-up period was 32 months (range, 1-87 months), and the median age of all patients was 66 years (age range, 34-90 years). Computed tomography (CT), MRI, PET/CT or PET/MRI were used for image examination. Among the 149 patients, 31 (20.8\%) developed PIF. The median age of these patients was 69 years (age range, 44-87 years). Univariate analysis using the log-rank test demonstrated that age ( $\geq 60$ years) was significantly associated with PIF. The median maximum standardized uptake value of PIF sites on PET/CT was 4.32 (range, 3.04-4.81), and that on PET/MRI was 3.97 (range, 1.21-5.96) $(\mathrm{P}=0.162)$. Notably, the detection time of PIF by PET/MRI was significantly earlier compared with PET/CT $(\mathrm{P}<0.05)$. The incidence of PIF after RT for cervical cancer was $20.8 \%$, and age was significantly associated with risk factors for such fractures. Taken together, these results suggest that PET/MRI, which offers the advantage of decreased radiation exposure to the patient, is useful for diagnosing PIF and can detect it earlier than PET/CT imaging.
\end{abstract}

Correspondence to: Professor Yoshito Terai, Department of Obstetrics and Gynecology, Kobe University Graduate School of Medicine, 7-5-1 Kusunoki-cho Chuo-ku, Kobe, Hyogo 650-0017, Japan

E-mail: yterai@med.kobe-u.ac.jp

Key words: uterine cervical neoplasms, radiotherapy, insufficiency fractures, positron-emission tomography, magnetic resonance imaging

\section{Introduction}

Radiotherapy (RT) is used to manage cervical cancer, either postoperatively or as a primary treatment (1), and major improvements in definitive RT have improved survival rates over the past 15-20 years (2). Pelvic insufficiency fracture (PIF) is a late complication of the therapy (3). According to a meta-analysis by Sapienza et al (4), the overall incidence of PIF is between $10-18 \%$, and the most common site for PIF is the sacrum. PIF can lead to intractable pain and limited mobility, which can seriously impact the quality of life of cervical cancer survivors (5). Risk factors associated with cervical cancer include age, postmenopausal period, low body weight and osteoporosis (5-14). Recently, intensity-modulated radiation therapy (IMRT) has been introduced to reduce acute and chronic gastrointestinal disorders (15). However, the most effective method in reducing PIF remains unclear.

Considering the increased number of cervical cancer survivors (16), it is important to clarify what factors increase the risk of PIF after RT for patients with cervical cancer, and detect PIF in the early phase before patients develop any symptoms. Most reports on PIF have used computed tomography (CT) and magnetic resonance imaging (MRI) as inspection methods (5-10,12-14,17-21). According to Lapina and Tiškevičius (22), the sensitivity and specificity in PIF diagnosis are 100 and $95.3 \%$ for MRI and 74.6 and $89.7 \%$ for CT, respectively. Recently, positron emission tomography (PET) is attracting great interest as an imaging test for the follow-up of cervical cancer recurrence (23). However, to the best of our knowledge, only a few reports have used PET $(5,11,19,21)$, and PET/MRI is yet to be investigated (4). The present study aimed to identify risk factors for PIF and investigate its incidence rate. In addition, the usefulness of PET/MRI in PIF diagnosis was assessed.

\section{Materials and methods}

Patient characteristics. The present study investigated 149 patients with cervical cancer who received definitive or adjuvant RT with/without concurrent chemotherapy between January 2013 and December 2018, and were followed up for more than one month after RT at Kobe University Hospital. Data collection ended in May 2020. The median follow-up 
period was 32 months (range, 1-87 months), and the median age of all patients was 66 years (age range, $34-90$ years). The histopathological type was squamous cell carcinoma (SCC) in 121 cases $(81.2 \%)$, adenocarcinoma in 26 cases $(17.4 \%)$, adenosquamous in one case $(0.7 \%)$ and clear cell carcinoma in one case $(0.7 \%)$. The median body mass index (BMI) was 21 (range, 14-40). A total of 27 cases (18.1\%) were in The International Federation of Gynecology and Obstetrics (FIGO) stage (24) was I, 62 cases in stage II (41.6\%), 40 cases in stage III (26.8\%) and 20 cases in stage IV (13.4\%). A total of 123 patients $(82.6 \%)$ received $\mathrm{RT}$ as a definitive treatment, $26(17.4 \%)$ received RT as postoperative adjuvant therapy, and $110(73.8 \%)$ received concurrent chemoradiotherapy. Table I presents patient characteristics, according to the presence or absence of PIF. Notably, no significant differences were observed between both groups.

The inclusion criteria were as follows: Patients whose medical records and prognosis could be obtained from medical records, and patients who had a definite diagnosis of cervical cancer and were followed up for at least one month after the end of radiotherapy. The exclusion criteria were as follows: Patients who were offered to not participate in this study based on publicly available information, and patients participating in or planning to participate in clinical studies involving drug therapy interventions.

Treatment. For definitive RT, external beam RT (EBRT) was performed using the four-field box technique, and intracavitary brachytherapy (ICBT) was subsequently performed using a remote after-loading system, in combination with EBRT, using the central shield by anteroposterior/posteroanterior field technique at $10 \mathrm{MV}$ photons. The upper limit of the standard irradiation field was the upper edge of the 5th lumbar vertebra, and the lower limit was at least $3 \mathrm{~cm}$ below the lower edge of the obturator foramen or the lower edge of the vaginal infiltration. The outer side of the anterior-posterior irradiation field was $1.5-2 \mathrm{~cm}$ outside the inner edge of the pelvis, and the anterior edge of the lateral irradiation field was $\sim 0.5 \mathrm{~cm}$ in front of the anterior pubic symphysis. A total dose of EBRT (TrueBeam $^{\mathrm{TM}}$; Varian Medical Systems) at 50.4 Gy (range, 40.0-50.4 Gy) and ICBT (Micro Selectron ${ }^{\mathrm{TM}}$; Nucletron BV) at 24 Gy (range, 18-30 Gy) was administered. For postoperative adjuvant RT, IMRT (TrueBeam ${ }^{\mathrm{TM}}$, Varian Medical Systems) was performed with a total dose of 50.4 Gy (range, 50.4-60.0 Gy). A total dose of 10.0 Gy (range, 3.6-16.2 Gy) lymph node boost (TrueBeam ${ }^{\mathrm{TM}}$; Varian Medical Systems) was given to 54 patients $(36.2 \%)$ with positive lymph node metastasis. For concurrent chemoradiotherapy, cisplatin (Cisplatin Maruko $^{\mathrm{TM}}$; Yakult Honsha Co., Ltd.) was administered once a week for $5-6$ cycles at $40 \mathrm{mg} / \mathrm{m}^{2}$.

Follow-up and diagnostic criteria of PIF. For cervical cancer, follow-up started 1 month after the end of radiation therapy, and in principle, it was continued for 5 years in the outpatient department. Follow-up was conducted every 1 to 3 months in the 1st and 2nd years, every 3 to 6 months in the 3rd year, and every 6 months in the 4 to 5 th years. Patients underwent imaging tests twice a year for the first 2 years and then annually thereafter. CT, MRI, PET/CT or PET/MRI were used as follow-up imaging methods. At the discretion of the two radiologists and two gynecologists, a recurrent check or follow-up was performed, and a CT, PET/CT or PET/MRI was selected. In addition, an MRI was selected depending on the symptoms, such as buttock or lower back pains. During the median follow-up period, 121 patients $(81.2 \%)$ underwent imaging with PET, while 66 patients $(44.3 \%)$ underwent imaging with PET/MRI. Patients who received multiple types of tests for the recurrence check or follow-up were excluded from the analysis.

The diagnostic criteria for PIF was as follows: CT for a fracture line or osteosclerosis without osteolytic changes, MRI for T1 hypointensity and T2 hyperintensity without a soft tissue mass, and PET for mild and diffuse fluorodeoxyglucose (FDG) accumulation. All images were diagnosed by two radiologists at Kobe University Hospital.

Statistical analysis. Statistical analysis was performed using $\mathrm{R}$ version 4.0.0 (https://cran.r-project.org/bin/windows/base/). For each factor in the fracture $(\mathrm{n}=31)$ and non-fracture groups $(n=118), \chi^{2}$ or Fisher's exact tests were used to assess the differences in frequency. The Mann-Whitney $U$ test was used to compare differences between continuous variables. Each exposure or each predictor was assessed using the log-rank test as univariate analysis. $\mathrm{P}<0.05$ was considered to indicate a statistically significant difference. This observational study complied with the STROBE guidelines (25).

\section{Results}

Incidence. Among the 149 patients investigated in the present study, 31 (20.8\%) developed PIF, and the median time for developing the condition was 17 months (range, 2-47 months). The median age of the fracture group was 69 years, of which 28 patients $(90.3 \%)$ were postmenopausal. The median age of the non-fracture group was 66 years, of which 94 patients $(79.7 \%)$ were postmenopausal. Notable, no significant differences in age and postmenopausal status were observed between the two groups. The median BMI was 20.9 in the fracture group and 21.0 in the non-fracture group, and the median number of deliveries was two in both the fracture and non-fracture groups, respectively. With regards to medical history, two patients $(6.5 \%)$ had diabetes, while three $(9.7 \%)$ had osteoporosis in the fracture group, whereas 15 patients $(12.7 \%)$ had diabetes, four $(3.4 \%)$ had rheumatic arthritis and $14(11.9 \%)$ had osteoporosis in the non-fracture group. There were no significant differences in medical history between the two groups. No patients received hormone replacement therapy (HRT) in the fracture group, while 10 patients received HRT in the non-fracture group. The histopathological types were 27 cases $(87.1 \%)$ of SCC, three $(9.7 \%)$ of adenocarcinoma and one $(3.2 \%)$ of adenosquamous in the fracture group, compared with 94 cases $(79.7 \%)$ of SCC, $23(19.5 \%)$ of adenocarcinoma and one $(0.8 \%)$ of clear cell carcinoma in the non-fracture group. There were no significant differences in histopathological types between the two groups. A total of two cases $(6.5 \%)$ were in FIGO stage I, 14 (45.2\%) in stage II, 13 (41.9\%) in stage III and two $(6.5 \%)$ in stage IV in the fracture group. Conversely, 25 cases $(21.2 \%)$ were in FIGO stage I, 48 $(40.7 \%)$ in stage II, $27(22.9 \%)$ in stage III and $18(15.3 \%)$ 
Table I. Patient characteristics.

\begin{tabular}{|c|c|c|c|}
\hline Characteristic & Fracture $(+)(n=31)$ & Fracture $(-)(\mathrm{n}=118)$ & P-value \\
\hline Median age (range), years & $69(44-87)$ & $66(34-90)$ & 0.0727 \\
\hline Median age of menopause (range), years & $50(40-57)$ & $50(40-60)$ & 0.3720 \\
\hline Menopausal status & & & 0.2938 \\
\hline Postmenopause & $28(90.3 \%)$ & $94(79.7 \%)$ & \\
\hline Premenopause & $2(6.5 \%)$ & $20(16.9 \%)$ & \\
\hline Unknown & $1(3.2 \%)$ & $4(3.4 \%)$ & \\
\hline Body mass index, $\mathrm{kg} / \mathrm{m}^{2}$ & $20.9(16.8-34.8)$ & $21.0(13.7-40.3)$ & 0.5858 \\
\hline Parity & $2(0-5)$ & $2(0-6)$ & 0.4134 \\
\hline \multicolumn{4}{|l|}{ Medical history } \\
\hline Diabetes mellitus & $2(6.5 \%)$ & $15(12.7 \%)$ & 0.5265 \\
\hline Rheumatoid arthritis & $0(0.0 \%)$ & $4(3.4 \%)$ & 0.5804 \\
\hline Osteoporosis & $3(9.7 \%)$ & $14(11.9 \%)$ & 1 \\
\hline Hormone replacement therapy & $0(0.0 \%)$ & $10(8.5 \%)$ & 0.1224 \\
\hline Histopathological type & & & 0.1571 \\
\hline Squamous cell carcinoma & $27(87.1 \%)$ & $94(79.7 \%)$ & \\
\hline Adenocarcinoma & $3(9.7 \%)$ & $23(19.5 \%)$ & \\
\hline Adenosquamous & $1(3.2 \%)$ & $0(0.0 \%)$ & \\
\hline Clear cell carcinoma & $0(0.0 \%)$ & $1(0.8 \%)$ & \\
\hline FIGO stage & & & 0.0552 \\
\hline $\mathrm{I}$ & $2(6.5 \%)$ & $25(21.2 \%)$ & \\
\hline II & $14(45.2 \%)$ & $48(40.7 \%)$ & \\
\hline III & $13(41.9 \%)$ & $27(22.9 \%)$ & \\
\hline IV & $2(6.5 \%)$ & $18(15.3 \%)$ & \\
\hline Treatment & & & 0.2129 \\
\hline Adjuvant CCRT & $0(0.0 \%)$ & $12(10.2 \%)$ & \\
\hline Adjuvant RT & $2(6.5 \%)$ & $12(10.2 \%)$ & \\
\hline Definitive CCRT & $22(71.0 \%)$ & $76(64.4 \%)$ & \\
\hline Definitive RT & $7(22.6 \%)$ & $18(15.3 \%)$ & \\
\hline ICBT & & & 0.2009 \\
\hline Yes & $28(90.3 \%)$ & $94(79.7 \%)$ & \\
\hline No & $3(9.7 \%)$ & $24(20.3 \%)$ & \\
\hline Lymph node boost & & & 1 \\
\hline Yes & $11(35.5 \%)$ & $43(36.4 \%)$ & \\
\hline No & $20(64.5 \%)$ & $75(63.6 \%)$ & \\
\hline IMRT & & & 0.1069 \\
\hline Yes & $2(6.5 \%)$ & $23(19.5 \%)$ & \\
\hline No & $29(93.5 \%)$ & $95(80.5 \%)$ & \\
\hline $\mathrm{PET} / \mathrm{CT}$ & & & 0.1561 \\
\hline Yes & $13(41.9 \%)$ & $68(57.6 \%)$ & \\
\hline No & $18(58.1 \%)$ & $50(42.4 \%)$ & \\
\hline PET/MRI & & & 0.6859 \\
\hline Yes & $15(48.4 \%)$ & $51(43.2 \%)$ & \\
\hline No & $16(51.6 \%)$ & $67(56.8 \%)$ & \\
\hline
\end{tabular}

FIGO, International Federation of Gynecology and Obstetrics; CCRT, computer-controlled radiation therapy; RT, radiotherapy; ICBT, intracavitary brachytherapy; IMRT, intensity-modulated radiation therapy; PET, positron emission tomography; CT, computed tomography; MRI, magnetic resonance imaging.

in stage IV in the non-fracture group. These results suggest that the FIGO stage was slightly higher in the fracture group compared with the non-fracture group $(\mathrm{P}=0.0552)$. In the facture group, no patients received adjuvant concurrent 
Table II. Characteristics of PIF.

\begin{tabular}{|c|c|c|c|c|c|c|c|}
\hline Patient & $\begin{array}{l}\text { Age, } \\
\text { years }\end{array}$ & $\begin{array}{l}\text { Time, } \\
\text { months }\end{array}$ & Site & Symptom & $\begin{array}{l}\text { Diagnostic } \\
\text { medium }\end{array}$ & $\begin{array}{l}\mathrm{PET} / \mathrm{CT} \\
\left(\mathrm{SUV}_{\max }\right)\end{array}$ & $\begin{array}{l}\text { PET/MRI } \\
\left(\mathrm{SUV}_{\max }\right)\end{array}$ \\
\hline 1 & 72 & 32 & Sacrum & Asymptomatic & $\mathrm{CT}$ & NA & NA \\
\hline 2 & 66 & 28 & Sacrum & Buttocks pain & $\mathrm{CT}$ & NA & NA \\
\hline 3 & 80 & 12 & Lumber spinal vertebrae & Buttocks pain & $\mathrm{PET} / \mathrm{CT}$ & 3.68 & NA \\
\hline 4 & 82 & 38 & Sacrum & Buttocks pain & $\mathrm{CT}$ & NA & NA \\
\hline 5 & 81 & 33 & Sacrumpubis & Buttocks pain & $\mathrm{CT}$ & NA & NA \\
\hline 6 & 76 & 25 & Sacrum & Asymptomatic & $\mathrm{PET} / \mathrm{CT}$ & 4.52 & NA \\
\hline 7 & 87 & 12 & Sacrum & Buttocks pain & MRI & NA & NA \\
\hline 8 & 61 & 15 & Sacrum & Buttocks pain & $\mathrm{PET} / \mathrm{CT}$ & 4.81 & NA \\
\hline 9 & 66 & 34 & Sacrumpubis & Asymptomatic & $\mathrm{PET} / \mathrm{CT}$ & 4.30 & NA \\
\hline 10 & 46 & 36 & Sacrumpubis & Buttocks pain & PET/MRI & NA & 5.96 \\
\hline 11 & 67 & 4 & Sacrum & Asymptomatic & $\mathrm{PET} / \mathrm{CT}$ & 4.11 & NA \\
\hline 12 & 67 & 47 & Sacrumpubis & Asymptomatic & $\mathrm{PET} / \mathrm{CT}$ & 4.81 & NA \\
\hline 13 & 51 & 22 & Sacrum & Asymptomatic & $\mathrm{CT}$ & NA & NA \\
\hline 14 & 58 & 14 & Sacrum & Buttocks pain & $\mathrm{CT}$ & NA & NA \\
\hline 15 & 69 & 7 & Sacrum & Buttocks pain & PET/MRI & NA & 4.06 \\
\hline 16 & 79 & 9 & Sacrumlumber spinal vertebrae & Asymptomatic & PET/MRI & NA & 4.02 \\
\hline 17 & 82 & 2 & Lumber spinal vertebrae & Buttocks pain & $\mathrm{CT}$ & NA & NA \\
\hline 18 & 60 & 32 & Sacrum & Buttocks pain & PET/MRI & NA & 1.21 \\
\hline 19 & 66 & 29 & Sacrum & Asymptomatic & PET/MRI & NA & 3.92 \\
\hline 20 & 69 & 13 & Sacrum & Asymptomatic & PET/MRI & NA & 5.23 \\
\hline 21 & 70 & 29 & Sacrumpubis & Asymptomatic & $\mathrm{CT}$ & NA & NA \\
\hline 22 & 75 & 15 & Sacrum & Asymptomatic & PET/MRI & NA & 2.50 \\
\hline 23 & 52 & 18 & Sacrum & Asymptomatic & $\mathrm{PET} / \mathrm{CT}$ & 3.04 & NA \\
\hline 24 & 74 & 7 & Lumber spinal vertebrae & Asymptomatic & $\mathrm{PET} / \mathrm{CT}$ & 4.27 & NA \\
\hline 25 & 73 & 23 & Sacrumpubis & Buttocks pain & $\mathrm{PET} / \mathrm{CT}$ & 4.33 & NA \\
\hline 26 & 44 & 17 & Sacrum & Asymptomatic & PET/MRI & NA & 3.39 \\
\hline 27 & 80 & 8 & Sacrumpubis & Asymptomatic & $\mathrm{CT}$ & NA & NA \\
\hline 28 & 62 & 21 & Sacrumpubis & Buttocks pain & PET/MRI & NA & 4.07 \\
\hline 29 & 76 & 6 & Sacrumpubis & Buttocks pain & $\mathrm{PET} / \mathrm{CT}$ & 4.34 & NA \\
\hline 30 & 67 & 8 & Sacrumthoracic spinal vertebrae & Asymptomatic & $\mathrm{CT}$ & NA & NA \\
\hline 31 & 56 & 8 & Sacrum & Asymptomatic & PET/MRI & NA & 3.88 \\
\hline
\end{tabular}

PIF, pelvic insufficiency fracture; PET, positron emission tomography; CT, computed tomography; MRI, magnetic resonance imaging; $\mathrm{SUV}_{\max }$, maximum standardized uptake value; NA, not available.

chemoradiotherapy (CCRT), two $(6.5 \%)$ received adjuvant RT, $22(71.0 \%)$ received definitive CCRT and seven $(22.6 \%)$ received definitive RT. Conversely, in the non-fracture group, 12 patients (10.2\%) received adjuvant CCRT, $12(10.2 \%)$ received adjuvant RT, $76(64.4 \%)$ received definitive CCRT and $18(15.3 \%)$ received definitive RT. A total of 28 patients $(90.3 \%)$ received ICBT, $11(35.5 \%)$ received a lymph node boost and two $(6.5 \%)$ received IMRT in the fracture group, whereas 94 patients $(79.7 \%)$ received ICBT, $43(36.4 \%)$ received a lymph node boost and $23(19.5 \%)$ received IMRT in the non-fracture group. Notably, no significant differences in the type of treatment were observed between the two groups. A total of 13 patients (41.9\%) had PET/CT as the imaging test, while $15(48.4 \%)$ had PET/MRI in the fracture group. Conversely, 68 patients $(57.6 \%)$ had PET/CT as the imaging test, while $51(43.2 \%)$ had PET/MRI in the non-fracture group. There were no significant differences in image examination between the two groups (Table I).

Sites. Table II presents the result of 31 patients who were diagnosed with PIF, 17 of which (54.8\%) were asymptomatic. A total of 28 patients has their fracture site in the sacrum, nine in the pubis and four in the lumber spinal vertebrae. A total of 11 patients $(35.5 \%)$ had multiple fractures (Table II), while 17 $(54.8 \%)$ were asymptomatic. Furthermore, 10 patients $(32.3 \%)$ were diagnosed with PIF on CT, one $(3.2 \%)$ on MRI, 10 $(32.3 \%)$ on PET/CT and 10 (32.3\%) on PET/MRI. In addition, $12 / 17$ patients $(70.6 \%)$ with asymptomatic PIF were diagnosed by PET-CT or PET-MRI.

Analysis of risk factors. The associations between PIF, patient characteristics and treatment related factors were 
Table III. Univariate analysis of risk factors for PIF.

\begin{tabular}{lcc}
\hline Variable & $95 \%$ CI & P-value \\
\hline Age, years $(\geq 60)$ & $47-N A$ & $<0.05$ \\
BMI, $\mathrm{kg} / \mathrm{m}^{2}(<21)$ & NA-NA & 0.505 \\
Postmenopausal status & NA-NA & 0.199 \\
Parity $(\geq 3)$ & $33-N A$ & 0.289 \\
SCC & NA-NA & 0.622 \\
FIGO stage $(\geq$ III $)$ & $33-N A$ & 0.0732 \\
ICBT & $36-N A$ & 0.438 \\
Lymph node boost & $36-N A$ & 0.188 \\
IMRT & NA-NA & 0.125 \\
Definitive radiotherapy & NA-NA & 0.0816
\end{tabular}

PIF, pelvic insufficiency fracture; CI, confidence interval; BMI, body mass index; SCC, squamous cell carcinoma; FIGO, International Federation of Gynecology and Obstetrics; ICBT, intracavitary brachytherapy; IMRT, intensity-modulated radiation therapy; NA, not available.

assessed (Table III). Univariate analysis using the log-rank test demonstrated that age ( $\geq 60$ years) was significantly associated with PIF $(\mathrm{P}<0.05)$. However, BMI $<21$, postmenopausal status, parity $\geq 3$, SCC, FIGO stage $\geq$ III, ICBT, a lymph node boost, IMRT and definitive RT were not significant risk factors for developing PIF.

Characteristics of PET/CT and PET/MRI in PIF diagnosis. The association between PIF diagnosis and the method of fracture detection, using either PET/CT or PET/MRI, was assessed. The median maximum standardized uptake value $\left(\mathrm{SUV}_{\max }\right)$ of PIF sites on PET/CT was 4.32 (range, 3.04-4.81) and that on PET/MRI was 3.97 (range, 1.21-5.96), which was not statistically significant $(\mathrm{P}=0.162$; Table II). Kaplan-Meier analysis was performed to compare patients who underwent image follow-up with PET/CT or PET/MRI during the median follow-up period, except for those who underwent both PET/CT and PET/MRI. The results demonstrated that the detection time of PIF by PET/MRI ( $n=40)$ was significantly earlier than that of PET/CT $(n=55)(P=0.0367$; Fig. 1$)$.

A diffuse linear FDG uptake. A diffuse linear FDG uptake was observed in parallel with the sacroiliac joint as a characteristic pattern of sacral fracture (Fig. 2). In addition, the features of FDG uptake were similar between PET/CT and PET/MRI in the present study (Fig. 2). The earliest MR sign, medullary edema, was seen and characterized as T1 hypointensity and T2 hyperintensity (Fig. 3).

\section{Discussion}

The results of the present study demonstrated that PET/MRI was useful for early diagnosis of recurrent cervical cancer, and for early detection of PIF compared with PET/CT. Currently, a few studies have investigated the incidence and risk factors of PIF via PET/CT as the imaging method; however, the effects of PET/MRI have not yet been investigated (5,26-28). To the

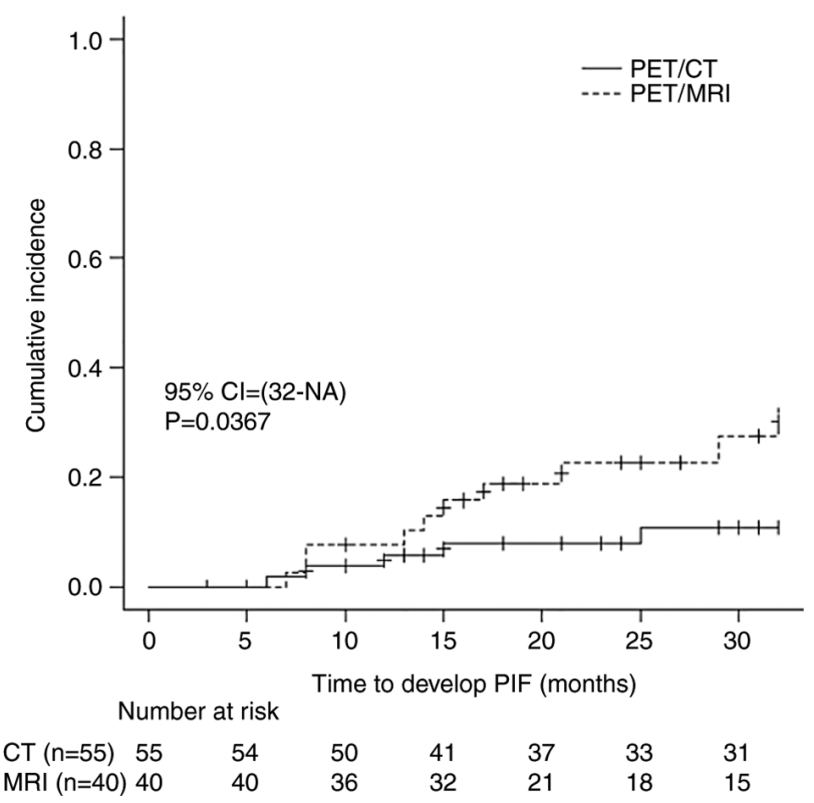

Figure 1. Comparison of the incidence of PIF in PET/CT and PET/MRI during the median follow-up period. The detection time of PIF by PET/MRI was significantly earlier than that by PET/CT. PIF, pelvic insufficiency fracture; PET, positron emission tomography; CT, computed tomography; MRI, magnetic resonance imaging; CI, confidence interval; NA, not available.

best of our knowledge, the present study was the first to report the incidence rate and risk factors of PIF via PET/MRI as the imaging method.

Radiation therapy of the pelvis can cause demineralization and decrease elastic resistance of the bone matrix (28). According to a recent meta-analysis, the overall incident rate of PIF was $14 \%$ (95\% confidence interval, 10-18\%, based on 21 studies) (4). The incidence rate of PIF in the present study was $20.8 \%$, which was slightly higher than the meta-analysis. Age has also been reported as a risk factor in several studies (5-11), as well as the present study. However, the results of the present study failed to exhibit significant differences in other risk factors between the groups. This may have been due to the small sample size used in the present study. Previous studies have identified risk factors for PIF, including postmenopausal status $(6-8,10,12)$, low body weight $(5,9-11)$ and osteoporosis $(13,14)$. In the present study, no significant differences were observed between the groups for some parameters such as menopausal status or BMI.

This study was a retrospective study and thus relies on medical records for information. However, data on the presence or absence of osteoporosis was insufficient and thus was not evaluated. Prospective studies are required to incorporate this data by screening patients prior to chemoradiotherapy. It has been reported that rheumatoid arthritis (RA) (12) and HRT (14) are risk factors for PIF. However, the present study was unable to assess the associations between PIF and RA or HRT as only a few patients had RA or had received HRT. Oh et al reported that a high radiation dose is a risk factor of PIF (11). At Kobe University Hospital, both EBRT and IMRT are performed during the treatment period in some cases and at the discretion of the radiation oncologist; therefore, it was not possible to calculate the total pelvic irradiation dose by simply adding the irradiation doses together in the present 
A

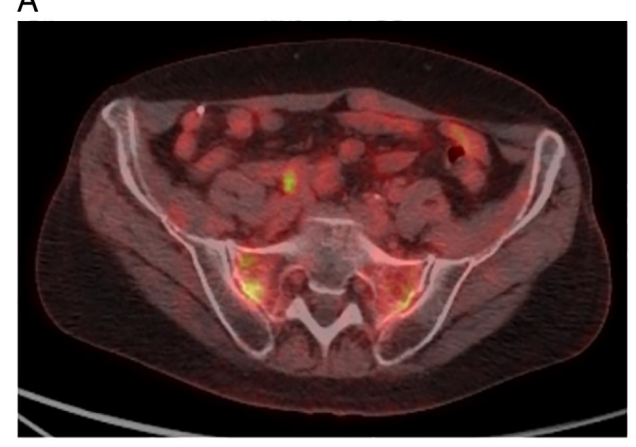

B

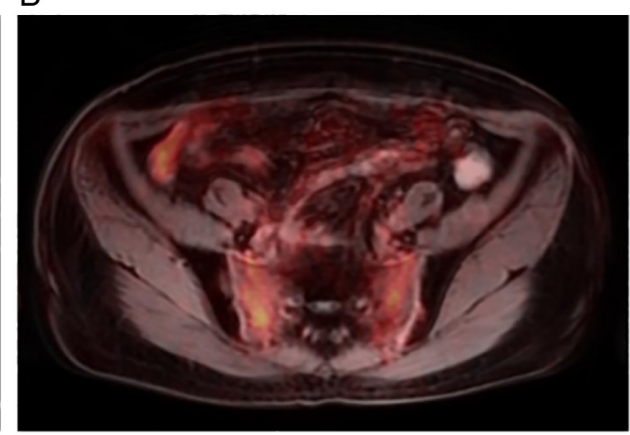

Figure 2. A diffuse linear FDG uptake parallel with the sacrum. The features of FDG uptake were similar between PET/CT and PET/MRI. (A) Patient 11 diagnosed by PET/CT (SUV ${ }_{\max }$ 4.11). (B) Patient 19 diagnosed by PET/MRI (SUV ${ }_{\max }$ 3.92). FDG, fluorodeoxyglucose; PET, positron emission tomography; $\mathrm{CT}$, computed tomography; MRI, magnetic resonance imaging; $\mathrm{SUV}_{\max }$, maximum standardized uptake value.

A

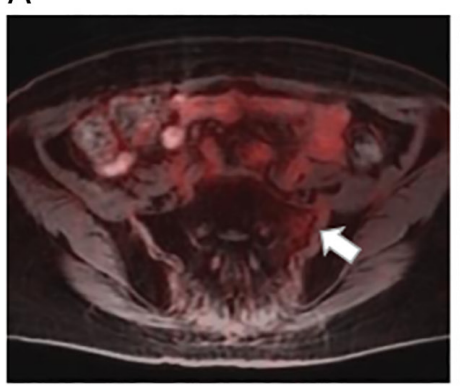

B

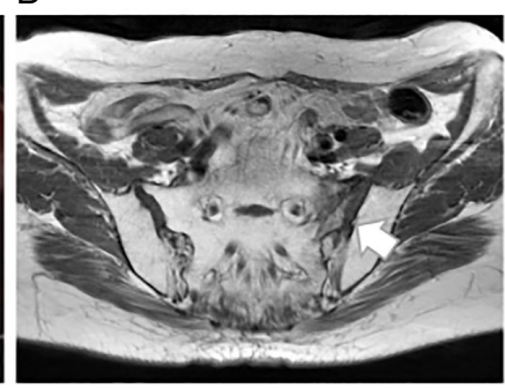

C

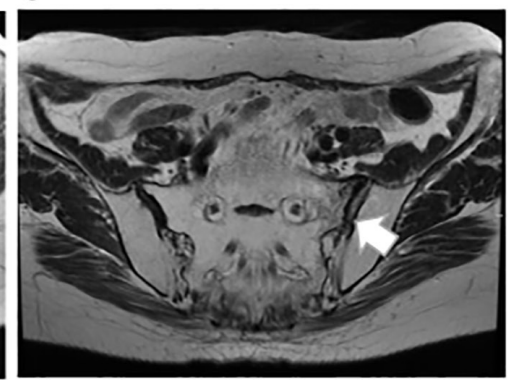

Figure 3. Earliest MR sign. The earliest MR sign, medullary edema, which is characterized as T1 hypointensity and T2 hyperintensity. (A) Patient 22 diagnosed by PET/MRI (SUV $\max _{2.5)}$ (B) T1-weighted image: Hypointensity and (C) T2-weighted image: Hyperintensity on the left side of the sacrum. PET, positron emission tomography; MRI, magnetic resonance imaging; $\mathrm{SUV}_{\max }$, maximum standardized uptake value.

study. Concurrent chemoradiotherapy is considered a risk factor of PIF; however, no previous studies have identified chemotherapy as a risk factor $(7,12,17)$, and neither did the present study. Ogino et al (29) reported that a history of three or more deliveries is a risk factor of PIF, but we did not find a significant difference in the present study. The sacrum is the predominant site of PIF and is reported to account for $73.6 \%$ of incidences (4). In the present study, fractures in the sacrum occurred in $27 / 31$ patients $(87.0 \%)$.

According to some reports, $43-77 \%$ of PIF cases are symptomatic, and patients experience pain that affects their quality of life $(5,30,31)$. Maintaining the quality of life for cancer survivors has become an important issue in recent years. It is even more important in patients with cervical cancer as it has a relatively young age of onset and has a long survival time when completely cured (32). According to the results of the present study, $45.2 \%$ of patients with PIF were symptomatic. Treatment is generally conservative, with analgesic administration and rehabilitation (33). Early diagnosis and treatment may improve pain, immobilization and thus mortality (33).

Imaging tests are rarely performed to solely diagnose PIF, and PIF is often found when checking the recurrence of cervical cancer. In previous reports, PET/CT was demonstrated to be more sensitive in detecting recurrence or metastasis compared with CT or MRI $(34,35) \mathrm{PET} / \mathrm{MRI}$ has been reported to exhibit comparable or better performance than PET/CT in detecting the recurrence of gynecologic cancer (36). Furthermore, PET/MRI has the advantage of lesion detection within the brain, breast, liver, kidneys, bone and pelvic lesion compared with PET/CT (37). In addition, PET/MRI offers the advantage of decreased radiation exposure compared with PET/CT (38). It is important for patients with cervical cancer to not only assess the detection of their recurrent disease but also to avoid the radiation-induced malignancies that can occur during a long follow-up period. Currently, several studies have reported on the usefulness of CT and MRI data in PIF diagnosis $(20,22,39)$, and according to Lapina and Tiškevičius (22), the sensitivity and specificity in PIF diagnosis are 100 and $95.3 \%$ for MRI and 74.6 and $89.7 \%$ for CT, respectively. Although the characteristics of PET/CT in PIF diagnosis have been previously reported $(5,27,28)$, their value remains unclear. In the present study, $54.8 \%$ of patients with PIF were asymptomatic, $70.6 \%$ of which were diagnosed by PET-CT or PET-MRI. Conversely, a previous report has indicated that only $39 \%$ of patients with asymptomatic PIF were diagnosed by CT or MRI (20).

It has been reported that low-grade FDG uptake is often observed at the fracture site (5). A diffuse linear FDG uptake is observed in parallel with the sacroiliac joint as a characteristic pattern of sacral fracture (Fig. 2). It is important to distinguish between benign and malignant fractures. The $\mathrm{SUV}_{\max }$ value and the FDG uptake pattern differ between malignant and benign fractures $(40,41)$. These reports are based on PET/CT and cannot be generally applied to PET/MRI; however, the results of the present study demonstrated no significant differences in the degree of $\mathrm{SUV}_{\max }$ between PET/CT and PET/MRI. In addition, the features of FDG uptake were 
similar between PET/CT and PET/MRI in the present study (Fig. 2). Furthermore, the results demonstrated that PET/MRI may detect PIF earlier than PET/CT (Fig. 1). This suggests that the detection time of PIF is earlier in MRI compared with CT (22). According to Grangier et al (42), the earliest MR sign, medullary edema, which is characterized as T1 hypointensity and T2 hyperintensity, is seen as early as 18 days after the onset of symptoms and persist as long as 516 days (Fig. 3). These findings suggest that PET/MRI imaging may detect PIF at an earlier stage before patients have developed symptoms compared with PET/CT, CT or MRI alone.

The present study is not without limitations. Given that this study was retrospective, there may be data that has not yet been revealed. Furthermore, the present study failed to conduct a questionnaire to determine whether patients had any symptoms, and only depended on medical records. Thus, patients may have had symptoms, even in cases that were considered asymptomatic. Furthermore, image follow-up was performed mainly for the purpose of checking the recurrence of cervical cancer and not for PIF examination. Given that the specific timing of image follow-up was left to the attending physician, there may have been some variation in the imaging timing for each patient. In addition, the attending physician decided which imaging medium to follow up with, and since it was not randomized, there may have been selection bias. Patients who had many images taken due to recurrence may have had PIF found earlier. There is also the possibility that $\mathrm{SUV}_{\max }$ may have had different values depending on the elapsed time after fracture. Furthermore, PIF may have been found secondarily during the recurrence check for cervical cancer, and it was not clear when it occurred. In addition, measured SUVs on $\mathrm{PET} / \mathrm{CT}$ and PET/MRI could be significantly different even in the same patient due to the difference in detectors and reconstruction methods. The present study did not include dose volume histogram. In some cases, both EBRT and IMRT were performed during the treatment period, and it was not possible to calculate the total pelvic irradiation dose simply by adding the irradiation doses. Therefore, it is possible that the prescribed dose was clarified by using the dose volume histogram. It has been reported that high irradiation dose is a risk factor for PIF (30). In the present study, it may have been possible to clarify whether the prescribed dose is a risk factor of developing PIF or not by analyzing the dose volume histogram.

In conclusion, the incidence of PIF after RT for cervical cancer was $20.8 \%$ in the present study. Age was significantly associated with the development of PIF. Furthermore, PET/MRI, which offers the advantage of decreased radiation exposure to the patient, may detect PIF at an earlier phase before patients develop symptoms, thus allowing them to undergo early counseling and treatment for bone health and to maintain a better quality of life after cervical cancer treatment. However, further trials and reports are required to establish the usefulness of PET/MRI in PIF cases.

\section{Acknowledgements}

The authors of the present study would like to thank Dr Sonoko Suda, Dr Keitaro Yamanaka, Dr Mamiko Sawada, Dr Masako Tomimoto, Dr Keiichi Washio, Dr Maho Shimizu, Dr Ryosuke
Takahashi, Dr Satoshi Nagamata and Dr Yuka Murata for collecting the data. They would also like to thank Dr Satoshi Senoh for technical advice on radiation therapy. These persons were affiliated with Kobe University Hospital.

\section{Funding}

No funding was received.

\section{Availability of data and materials}

The datasets used and/or analyzed during the current study are available from the corresponding author upon reasonable request.

\section{Authors' contributions}

MA, MM, KS participated in data collection and analyzed and interpreted patient data. RS, YU nad MN analyzed and interpreted the radiological data. YT and MM confirm the authenticity of all the raw data. YT contributed to the study conception and design. MA and YT drafted the manuscript. All authors have read and approved the final manuscript.

\section{Ethics approval and consent to participate}

The present study was approved by Kobe University Hospital Clinical and Translational Research Center (IRB no. B200206, October 23rd, 2020). Given that this study was an observational study using existing data, patient consent was waived. All patient data were analyzed retrospectively, and no tissues were examined. However, all study details were disclosed to patients, and they were given the option to refuse registration via e-mail.

\section{Patient consent for publication}

Not applicable.

\section{Competing interests}

The authors declare that they have no competing interests.

\section{References}

1. Klaitong C, Meannuch E, Kaewbunperm U, Klaiphibule P and Sinthusek T: EP-1512: Radiotherapy at pelvis region in menopausal cervix cancer induce osteopenia/osteoporosis. Radiother Oncol 127 (Suppl 1): S819-S820, 2018.

2. Small W Jr and Kachnic L: Postradiotherapy pelvic fractures cause for concern or opportunity for future research? JAMA 294 : 2635-2637, 2005.

3. Viswanathan AN, Lee LJ, Eswara JR, Horowitz NS, Konstantinopoulos PA, Mirabeau-Beale KL, Rose BS, Von Keudell AG and Wo JY: Complications of pelvic radiation in patients treated for gynecologic malignancies. Cancer 120: 3870-3883, 2014.

4. Sapienza LG, Salcedo MP, Ning MS, Jhingran A, Klopp AH, Calsavara VF, Schmeler KM, Leite Gomes MJ, de Freitas Carvalho E and Baiocchi G: Pelvic insufficiency fractures after external beam radiation therapy for gynecologic cancers: A meta-analysis and meta-regression of 3929 patients. Int J Radiat Oncol Biol Phys 106: 475-484, 2020.

5. Park S, Kim J, Lee J and Park IK: Pelvic insufficiency fracture after radiotherapy in patients with cervical cancer in the era of PET/CT. Radiat Oncol J 29: 269-276, 2011. 
6. Ramlov A, Pedersen EM, Røhl L, Worm E, Fokdal L, Lindegaard JC and Tanderup K: Risk factors for pelvic insufficiency fractures in locally advanced cervical cancer following intensity modulated radiation therapy. Int J Radiat Oncol Biol Phys 97: 1032-1039, 2017

7. Bazire L, Xu H, Foy JP, Amessis M, Malhaire C, Cao K, De La Rochefordiere A and Kirova YM: Pelvic insufficiency fracture (PIF) incidence in patients treated with intensity-modulated radiation therapy (IMRT) for gynaecological or anal cancer: Single-institution experience and review of the literature. Br J Radiol 90: 20160885, 2017.

8. Cai ZX, Li Y, Yang ZH, Gong S, Zhang MD and Chen JY: MRI and associated clinical characteristics of pelvic insufficiency fracture in cervical carcinoma patients after radiation therapy. Chinese J Med Imaging Technol 31: 1483-1486, 2015.

9. Tokumaru S, Toita T, Oguchi M, Ohno T, Kato S, Niibe Y, Kazumoto T, Kodaira T, Kataoka M, Shikama N, et al: Insufficiency fractures after pelvic radiation therapy for uterine cervical cancer: An analysis of subjects in a prospective multi-institutional trial, and cooperative study of the Japan radiation oncology group (JAROG) and Japanese radiation oncology Study Group (JROSG). Int J Radiat Oncol Biol Phys 84 e195-e200, 2012.

10. Schmeler KM, Jhingran A, Iyer RB, Sun CC, Eifel PJ, Soliman PT, Ramirez PT, Frumovitz M, Bodurka DC and Sood AK: Pelvic fractures after radiotherapy for cervical cancer: Implications for survivors. Cancer 116: 625-630, 2010.

11. OhD,Huh SJ,Park W,Ju SG, Nam H and Lee JE: Clinical outcomes in cervical cancer patients treated by FDG-PET/CT-based 3 -dimensional planning for the first brachytherapy session. Medicine (Baltimore) 95: e3895, 2016.

12. Yamamoto K, Nagao S, Suzuki K, Kogiku A, Senda T, Yano H, Kitai M, Shiozaki T, Matsuoka K and Yamaguchi S: Gynecologic Oncology Pelvic fractures after de fi nitive and postoperative radiotherapy for cervical cancer: A retrospective analysis of risk factors. Gynecol Oncol 147: 585-588, 2017.

13. Weidenbacher B, Borm K and Oechsner M: Pelvic fractures after radiotherapy for cervical cancer. Strahlenther Onkol 194, 2018.

14. Shih KK, Folkert MR, Kollmeier MA, Abu-Rustum NR, Sonoda Y, Leitao MM, Barakat RR and Alektiar KM: Pelvic insufficiency fractures in patients with cervical and endometrial cancer treated with postoperative pelvic radiation. Gynecol Oncol 128: 540-543, 2013.

15. Ta L, Jt G, Se D, Cc H and Hjn A: Effects of pelvic radiotherapy for primary pelvic cancers (Review). Cochrane Database Syst Rev, 2018.

16. Elit L and Reade CJ: Recommendations for follow-up care for gynecologic cancer survivors. Obstet Gynecol 126: 1207-1214, 2015.

17. Mehmood Q, Beardwood M, Swindell R, Greenhalgh S, Wareham T, Barraclough L, Livsey J and Davidson SE: Insufficiency fractures in patients treated with pelvic radiotherapy and chemotherapy for uterine and cervical cancer. Eur J Cancer Care (Engl) 23: 43-50, 2014.

18. Kuji S, Hirashima Y, Komeda S, Tanaka A, Abe M, Takahashi N, Takekuma M, Asakura H, Harada H and Nishimura T: Feasibility of extended-field irradiation and intracavitary brachytherapy combined with weekly cisplatin chemosensitization for IB2-IIIB cervical cancer with positive paraaortic or high common iliac lymph nodes: A retrospective review. Int J Clin Oncol 19 341-347, 2014.

19. Jung J, Park G and Kim YS: Definitive extended-field intensity-modulated radiotherapy with chemotherapy for cervical cancer with para-aortic nodal metastasis. Anticancer Res 34: 4361-4366, 2014

20. Uezono H, Tsujino K, Ota Y, Nagano F, Soejima T, Moriki K and Sasaki R: Pelvic insufficiency fracture after definitive radiotherapy for uterine cervical cancer: Retrospective analysis of risk factors. J Radiat Res 54: 1102-1109, 2013.

21. Kim J, Lee KJ, Park KR, Ha B, Kim YJ, Jung W, Lee R, Kim SC, Moon HS, Ju W, et al: Treatment outcomes after adjuvant radiotherapy following surgery for patients with stage I endometrial cancer. Radiat Oncol J 34: 265-272, 2016

22. Lapina $\mathrm{O}$ and Tiškevičius S: Sacral insufficiency fracture after pelvic radiotherapy: A diagnostic challenge for a radiologist. Med 50: 249-254, 2014

23. NCCN clinical practice guidelines in oncology. Cervical Cancer Version 4. Natl Compr Cancer Netw, 2019.

24. Pecorelli S, Zigliani L and Odicino F: Revised FIGO staging for carcinoma of the cervix. Int J Gynecol Obstet 105: 107-108, 2009
25. Von Elm E, Altman DG, Egger M, Pocock SJ, Gøtzsche PC and Vandenbrouckef JP; STROBE Initiative: The Strengthening the Reporting of Observational Studies in Epidemiology (STROBE) Statement: Guidelines for reporting observational studies. Bull World Health Organ 85: 867-872, 2007.

26. Halaç M, Mut SS, Sönmezoglu K, Ylmaz MH, Ozer H and Uslu I: Avoidance of misinterpretation of an FDG positive sacral insufficiency fracture using PET/CT scans in a patient with endometrial cancer: A case report. Clin Nucl Med 32: 779-781, 2007

27. Salavati A, Shah V, Wang ZJ, Yeh BM, Costouros NG and Coakley FV: F-18 FDG PET/CT findings in postradiation pelvic insufficiency fracture. J Clin Imaging 35: 139-142, 2011.

28. Chung YK, Lee YK, Yoon BH, Suh DH and Koo KH: Pelvic insufficiency fractures in cervical cancer after radiation therapy: A meta-analysis and review. In Vivo (Brooklyn) 35: 1109-1115, 2021.

29. Ogino I, Okamoto N, Ono Y, Kitamura T and Nakayama $\mathrm{H}$ : Pelvic insufficiency fractures in postmenopausal woman with advanced cervical cancer treated by radiotherapy. Radiother Oncol 68: 61-67, 2003

30. Kwon JW, Huh SJ, Yoon YC, Choi SH, Jung JY, Oh D and Choe BK: Pelvic bone complications after radiation therapy of uterine cervical cancer: Evaluation with MRI. AJR Am J Roentgenol 191: 987-994, 2008.

31. Kido A, Yoshida S, Shimoda E, Ishida Y, Hasegawa M, Kobayashi H, Honoki K, Horikawa H and Tanaka Y: Walking disability in patients with pelvic insufficiency fracture after radiotherapy for uterine cervical cancer. Prog Rehabil Med 1: 20160009,2016

32. Thapa N, Maharjan M, Xiong Y, Jiang D, Nguyen TP, Petrini MA and Cai H: Impact of cervical cancer on quality of life of women in Hubei, China. Sci Rep 8: 11993, 2018.

33. van den Blink QU, Garcez K, Henson CC, Davidson SE and Higham CE: Pharmacological interventions for the prevention of insufficiency fractures and avascular necrosis associated with pelvic radiotherapy in adults. Cochrane Database Syst Rev 4: CD010604, 2018.

34. Choi HJ, Ju W, Myung SK and Kim Y: Diagnostic performance of computer tomography, magnetic resonance imaging, and positron emission tomography or positron emission tomography/computer tomography for detection of metastatic lymph nodes in patients with cervical cancer: Meta-analysis. Cancer Sci 101: 1471-1479, 2010.

35. Pantola S, Kala S, Kala C, Sampath S and Shukla M: Comparative study of positron emission tomography/computed tomography and computed tomography in the evaluation of post-treatment carcinoma cervix patients. Indian J Nucl Med 33: 194-201, 2018

36. Broski SM, Goenka AH, Kemp BJ and Johnson GB: Clinical PET/MRI: 2018 update. AJR Am J Roentgenol 211: 295-313, 2018.

37. Rosenkrantz AB, Friedman K, Chandarana H, Melsaether A, Moy L, Ding YS, Jhaveri K, Beltran L and Jain R: Current status of hybrid PET/MRI in oncologic imaging. Am J Roentgenol 206: $162-172,2016$.

38. Schwartz M, Gavane SC, Bou-Ayache J, Kolev V, Zakashansky K, Prasad-Hayes M, Taouli B, Chuang L and Kostakoglu L: Feasibility and feasibility and diagnostic performance of hybrid pet/mri compared with pet/ct for gynecological malignancies: A prospective pilot study. Abdom Radiol 43: 3462-3467, 2018.

39. Ugurluer G, Akbas T, Arpaci T, Ozcan N and Serin M: Bone complications after pelvic radiation therapy: Evaluation with MRI. J Med Imaging Radiat Oncol 58: 334-340, 2014.

40. He X, Zhao L, Guo X, Zhao L, Wu J, Huang J, Sun L, Xie C and Chen H: Differential diagnostic value of 18 F-FDG PET/CT for benign and malignant vertebral compression fractures: Comparison with magnetic resonance imaging. Cancer Manag Res 10: 2105-2115, 2018.

41. Mauch JT, Carr CM, Cloft H and Diehn FE: Review of the imaging features of benign osteoporotic and malignant vertebral compression fractures. AJNR Am J Neuroradiol 39: 1584-1592, 2018.

42. Grangier C, Garcia J, Howarth NR, May M and Rossier P: Role of MRI in the diagnosis of insufficiency fractures of the sacrum and acetabular roof. Skeletal Radiol 26: 517-524, 1997.

This work is licensed under a Creative Commons Attribution-NonCommercial-NoDerivatives 4.0 International (CC BY-NC-ND 4.0) License. 